\title{
Effect of the solvent on the mechanical and structural properties of $\mathrm{N}$-alkyldiamide organogels
}

Leïla Khacef ${ }^{a, *}$, Philippe Legros ${ }^{b}$, Pascal Hervé ${ }^{a}$, Guillaume Ovarlez ${ }^{a}$ and Yaocihuatl Medina-Gonzalez ${ }^{a}$

aUniversity of Bordeaux, CNRS, Solvay, LOF, UMR 5258, 33608 Pessac, France

bUniversity of Bordeaux, CNRS, PLACAMAT, UMS3626, 33608 Pessac, France

*Corresponding author. E-mail : Leila.khacef-ext@solvay.com

\section{Supplementary Information}




\section{General procedure for the gelator C18' synthesis}

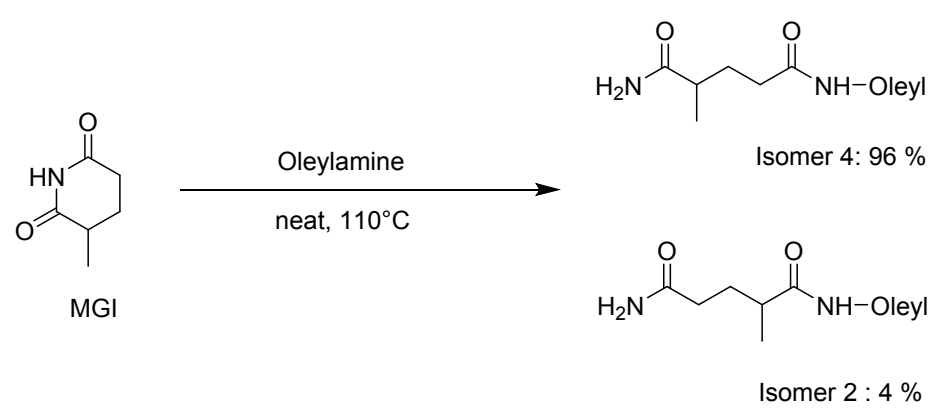

Figure S1: Gelator C18' synthesis using MGI precursor and oleylamine.

In a $1 \mathrm{~L}$ round bottom flask equipped with a mechanical stirrer and a condenser, $63,57 \mathrm{~g}$ of methylglutarimide (MGI) (0.5 mol, 1eq.) and $127.06 \mathrm{~g}$ of oleylamine $(0,475 \mathrm{~mol}, 0.95$ eq.) were added. The suspension was heated at $110^{\circ} \mathrm{C}$ during six hours. The mixture was then cooled down to $80^{\circ} \mathrm{C}$, and $350 \mathrm{~mL}$ of methylethylketone were added. The mixture was then let to cool down to room temperature, leading to a crystallization. The mixture was filtered and the solid was washed twice with $100 \mathrm{~mL}$ of ethyl acetate. The solid was then suspended in $1 \mathrm{~L}$ of methylethylketone, and the mixture was heated at $80^{\circ} \mathrm{C}$ then filtered. The solid of interest was recovered in the filtrate after crystallization at room temperature. The obtained solid (12 g, 19\% yield) contained an isomer 4/ isomer 2 ratio of 96/4 (calculated by NMR). Each isomer holds an asymmetrical carbon, leading to two enantiomers. However, the enantiopurity was not controlled and we hypothesized that the final product is a racemate gelator.

\section{Gelification trials}

\section{Gelification procedure:}

A list of 67 solvents was created and gathered solvents that can be heated up to $100-120^{\circ} \mathrm{C}$ (solvents with high enough boiling points). The solvent list is available in the following table (Table 1). The formulation of the gelator and the solvent was made according to the procedure described in the article. First, the formulations were stirred at room temperature for $24 \mathrm{~h}$, then all the insoluble formulations were heated at different temperature levels $\left(50^{\circ} \mathrm{C}, 80^{\circ} \mathrm{C}\right.$ and $\left.120^{\circ} \mathrm{C}\right)$ until the mixtures reached solubilization which was assessed by a visual control. After cooling down to room temperature (30 min), the formulations were classified into four categories: "Solutions', "Precipitates", "Transparent gels", "Turbid gels". The classification of the formulations was determined by a visual observation. Because of this qualitative assessment of the gelification, some ambiguous formulations were detected, especially when some formulations seem to consist of a swollen solid. That is why the fourth category "Turbid gels" was added. However, the lack of homogeneity in the formulation (presence of seeds for example) is the major criterion to classify a formulation as a "Precipitate".

Table S1: Solvent list for the gelification trials with regards to their HSP. The results of the gelification tests for each solvent are displayed in the "classification" column.

\begin{tabular}{|c|c|c|c|c|c|}
\hline Solvant & $\boldsymbol{\delta} \mathbf{d}\left(\mathbf{M P a}^{\mathbf{0 . 5}}\right)$ & $\boldsymbol{\delta} \mathbf{p}\left(\mathbf{M P a}^{\mathbf{0 . 5}}\right)$ & $\mathbf{\delta h}\left(\mathbf{M P a}^{\mathbf{0 . 5}}\right)$ & Solvent class & Classification \\
\hline Diacetone Alcohol & 15.8 & 8.2 & 10.8 & Alcohol & Precipitates \\
\hline Water & 15.5 & 16 & 42.3 & Alcohol & Precipitates \\
\hline
\end{tabular}




\begin{tabular}{|c|c|c|c|c|c|}
\hline Formamide & 17.2 & 26.2 & 19 & Amide & Precipitates \\
\hline $\begin{array}{c}\text { Dimethylformamide } \\
\text { (DMF) }\end{array}$ & 17.4 & 13.7 & 11.3 & Amide & Precipitates \\
\hline Butylene carbonate & 17.5 & 9.8 & 3 & Carbonate & Precipitates \\
\hline Propylene Carbonate & 20 & 18 & 4.1 & Carbonate & Precipitates \\
\hline Propylene Glycol & 16.8 & 10.4 & 21.3 & Diol & Precipitates \\
\hline 1-4-butanediol & 16.6 & 11 & 20.9 & Diol & Precipitates \\
\hline GBL & 18 & 16.6 & 7.4 & Ester & Precipitates \\
\hline Triacetin & 16.5 & 4.5 & 9.1 & Ester & Precipitates \\
\hline Butyl acetate & 15.8 & 3.7 & 6.3 & Ester & Precipitates \\
\hline Cyclohexanone & 17.8 & 8.4 & 5.1 & Ketone & Precipitates \\
\hline $\begin{array}{l}\text { Methyl ethyl ketone } \\
\text { (MEK) }\end{array}$ & 16 & 9 & 5.1 & Ketone & Precipitates \\
\hline Acetophenone & 18.8 & 9 & 4 & Ketone & Precipitates \\
\hline Benzyl Alcohol & 18.4 & 6.3 & 13.7 & Alcohol & Solutions \\
\hline 1-Butanol & 16 & 5.7 & 15.8 & Alcohol & Solutions \\
\hline Methanol & 14.7 & 12.3 & 22.3 & Alcohol & Solutions \\
\hline Isooctyl Alcohol & 14.4 & 7.3 & 12.9 & Alcohol & Solutions \\
\hline Isopropanol & 15.8 & 6.1 & 16.4 & Alcohol & Solutions \\
\hline 2-butanol & 15.8 & 5.7 & 14.5 & Alcohol & Solutions \\
\hline 1-octanol & 16 & 5 & 11.2 & Alcohol & Solutions \\
\hline 1-nonanol & 16 & 4.8 & 11 & Alcohol & Solutions \\
\hline Ethanol & 15.8 & 8.8 & 19.4 & Alcohol & Solutions \\
\hline 1-decanol & 16 & 4.7 & 10.5 & Alcohol & Solutions \\
\hline N.N-Diethyl Acetamide & 16.4 & 11.3 & 7.5 & Amide & Solutions \\
\hline $\begin{array}{l}\text { N-methyl pyrrolidone } \\
\text { (NMP) }\end{array}$ & 18 & 12.3 & 7.2 & Amide & Solutions \\
\hline N.N-Dimethyl Acetamide & 16.8 & 11.5 & 9.4 & Amide & Solutions \\
\hline Acetic acid & 14.5 & 8 & 13.5 & Carboxylic acid & Solutions \\
\hline Hexanoic acid & 16.3 & 4.2 & 11.5 & Carboxylic acid & Solutions \\
\hline 2-methylbutyric acid & & & & Carboxylic acid & Solutions \\
\hline $\begin{array}{l}\text { Methyl Isobutyl Ketone } \\
\text { (MIBK) }\end{array}$ & 15.3 & 6.1 & 4.1 & Ketone & Solutions \\
\hline Cyclohexane & 16.8 & 0 & 0.2 & Alkane & Transparent gels \\
\hline Hexadecane & 16.3 & 0 & 0 & Alkane & Transparent gels \\
\hline Decane & 15.7 & 0 & 0 & Alkane & Transparent gels \\
\hline Dodecane & 16 & 0 & 0 & Alkane & Transparent gels \\
\hline Tetradecane & 15.7 & 0 & 0 & Alkane & Transparent gels \\
\hline Bicyclohexyl & 17 & 0 & 0 & Alkane & Transparent gels \\
\hline Dodecylbenzene & 16.7 & 1.1 & 1.8 & Alkyl benzene & Transparent gels \\
\hline Decylbenzene & 16.8 & 1.4 & 2.1 & Alkyl benzene & Transparent gels \\
\hline Nonylbenzene & 16.9 & 1.4 & 2.1 & Alkyl benzene & Transparent gels \\
\hline Octylbenzene & 16.9 & 1.4 & 2.1 & Alkyl benzene & Transparent gels \\
\hline Butylbenzene & 17.4 & 0.1 & 1.1 & Alkyl benzene & Transparent gels \\
\hline Pentylbenzene & 17.2 & 1.8 & 2.5 & Alkyl benzene & Transparent gels \\
\hline Hexylbenzene & 17.4 & 1.5 & 2.3 & Alkyl benzene & Transparent gels \\
\hline Heptylbenzene & 17 & 1.8 & 2.3 & Alkyl benzene & Transparent gels \\
\hline Toluene & 18 & 1.4 & 2 & $\begin{array}{c}\text { Aromatic } \\
\text { hydrocarbon }\end{array}$ & Transparent gels \\
\hline Tetrahydronaphtalene & 19.6 & 2 & 2.9 & $\begin{array}{c}\text { Aromatic } \\
\text { hydrocarbon }\end{array}$ & Transparent gels \\
\hline o-xylene & 17.8 & 1 & 3.1 & $\begin{array}{c}\text { Aromatic } \\
\text { hydrocarbon }\end{array}$ & Transparent gels \\
\hline Mesitylene & 18 & 0.6 & 0.6 & $\begin{array}{c}\text { Aromatic } \\
\text { hydrocarbon }\end{array}$ & Transparent gels \\
\hline
\end{tabular}




\begin{tabular}{|c|c|c|c|c|c|}
\hline m-xylene & 18 & 2.3 & 2.3 & $\begin{array}{c}\text { Aromatic } \\
\text { hydrocarbon }\end{array}$ & Transparent gels \\
\hline Methyl salicylate & 18.1 & 8 & 13.9 & $\begin{array}{c}\text { Beta-hydroxy } \\
\text { ester }\end{array}$ & Transparent gels \\
\hline Ethyl salicylate & 18.2 & 8.1 & 9.3 & $\begin{array}{l}\text { Beta-hydroxy } \\
\text { ester }\end{array}$ & Transparent gels \\
\hline Benzyl salicylate & 19.1 & 8.2 & 11 & $\begin{array}{c}\text { Beta-hydroxy } \\
\text { ester }\end{array}$ & Transparent gels \\
\hline Benzyl acetate & 18.3 & 5.7 & 6 & Ester & Transparent gels \\
\hline Chlorobenzene & 19 & 4.3 & 1.9 & $\begin{array}{c}\text { Halogenated } \\
\text { aromatic }\end{array}$ & Transparent gels \\
\hline Tetrachloroethylene & 18.3 & 5.7 & 0 & $\begin{array}{c}\text { Halogenated } \\
\text { aromatic }\end{array}$ & Transparent gels \\
\hline o-dichlorobenzene & 19.2 & 6.3 & 3.3 & $\begin{array}{c}\text { Halogenated } \\
\text { aromatic }\end{array}$ & Transparent gels \\
\hline Glycerol carbonate & 17.9 & 25.5 & 17.4 & Carbonate & Turbid gels \\
\hline Ethylene glycol & 17 & 11 & 26 & Diol & Turbid gels \\
\hline Diethylene glycol & 16.6 & 12 & 19 & Diol & Turbid gels \\
\hline Propyl acetate & 15.3 & 4.3 & 7.6 & Ester & Turbid gels \\
\hline Tert-butyl Acetate & 15 & 3.7 & 6 & Ester & Turbid gels \\
\hline Octyl acetate & 15.8 & 2.9 & 5.1 & Ester & Turbid gels \\
\hline Amyl acetate & 15.8 & 3.3 & 6.1 & Ester & Turbid gels \\
\hline Hexyl acetate & 15.8 & 2.9 & 5.9 & Ester & Turbid gels \\
\hline Decyl acetate & 16.1 & 3.1 & 4 & Ester & Turbid gels \\
\hline 1-bromonaphthalene & 20.6 & 3.1 & 4.1 & $\begin{array}{c}\text { Halogenated } \\
\text { aromatic }\end{array}$ & Turbid gels \\
\hline
\end{tabular}

\section{Gelification results:}

The results were analyzed according to the Hansen Solubility Parameters. The formulations with regards to their classification were plotted in the Hansen 3D space ( $\delta \mathrm{d}, \delta \mathrm{p}, \delta \mathrm{h}$ ) (Figure 2 ) in order to identify relevant clusters of respectively "Solutions", "Precipitates", "Turbid gels" and "Transparent gels" formulations. The Figure 3 gives 2D projections of the Hansen space for clarity. Even though the borders between the domains are not clear, we can have a clue about the behavior of the solvents. Indeed, the solubility domain seems to be clustered for mid-polar and mid-protic solvents typically primary alcohol solvents. However, when increasing the polarity and the proticity of the solvent, the $\mathrm{N}$-oleyldiamide becomes insoluble in those solvents because of the oleyl chain. On the other hand, apolar and aprotic solvents lead also to the oleyldiamide precipitation at room temperature because of the diamide part of the molecule which is totally insoluble in those solvents at room temperature. When those formulations are heated and then cooled down gels may be formed. We hypothesized that in the solvents where gels are formed, the affinity of the gelator C18' with the solvent is perfectly balanced, the oleyl chain has a strong affinity with apolar solvents such as alkanes or aromatics on the other hand in those solvents the diamide self-assembly through $\mathrm{H}$-bonding is not disturbed by competitive $\mathrm{H}$-bond interactions due to the solvent. At the moment, no clear distinction has been made between "turbid gels", "transparent gels" and "precipitates". For this reason, this analysis has enabled us to select interesting solvents for further investigation. In the class "transparent gels", we have selected Aromatics, n-Alkanes, Esters and Beta-Hydroxy esters. In the class "Turbid gels", Glycerol carbonate and Ethylene glycol have been chosen. 


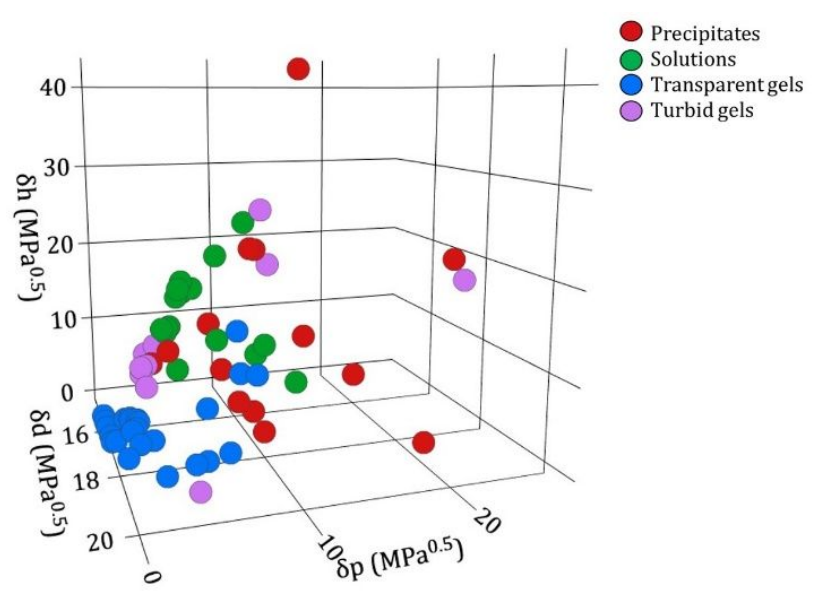

Figure S2: Representation of the gelification trials in the Hansen 3D space.

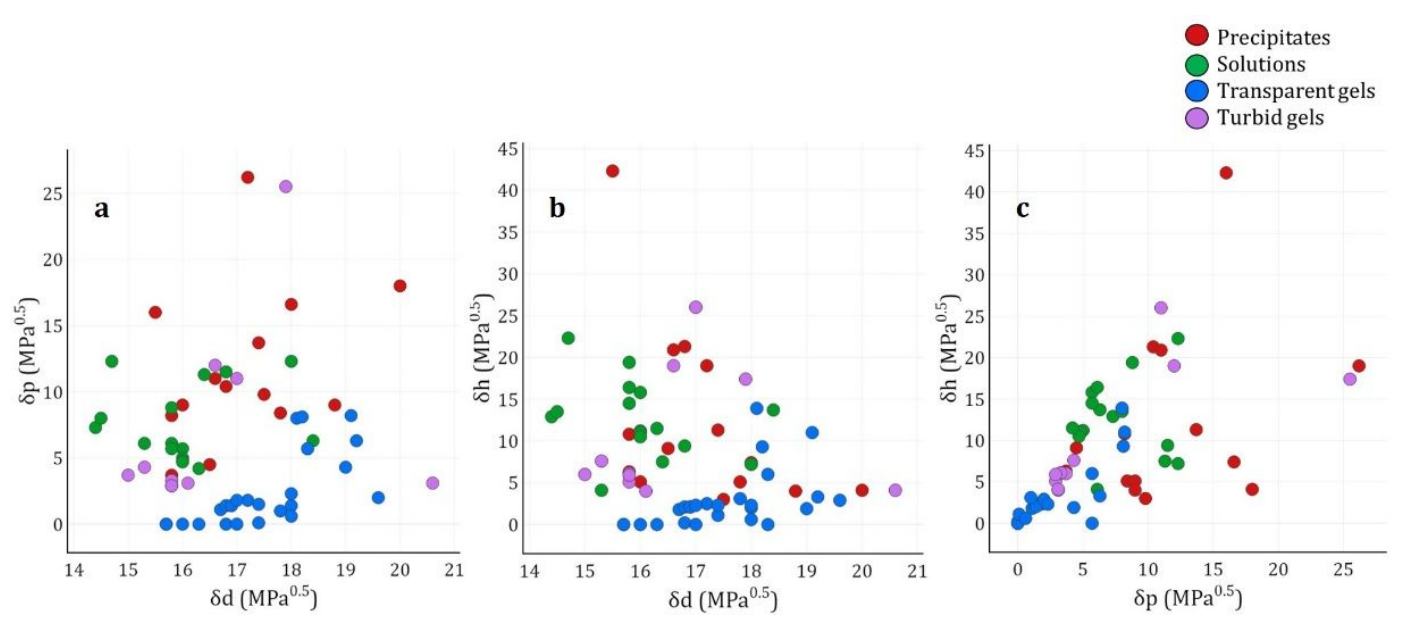

Figure S3: 2D projections of the gelification results in the Hansen space according to a) the $\delta d / \delta p$ plan, b) $\delta d / \delta h$ plan and c) the $\delta p / \delta h$ plan.

\section{Cryo-SEM measurements}

$\underline{\text { Temperature and Pressure values during solvent sublimation: }}$

The following table presents the values of temperature and pressure during the sublimation of selected solvents. These values are indicative and were measured approximately.

Table S2: Temperature and Pressure obtained during the sublimation of selected solvents.

\begin{tabular}{|c|c|c|}
\hline Solvent & $\begin{array}{c}\text { Range of sublimation } \\
\text { temperatures }\left({ }^{\circ} \mathrm{C}\right)\end{array}$ & $\begin{array}{c}\text { Antechamber pressure } \\
(\mathrm{mbar})\end{array}$ \\
\hline o-xylene & {$[-69 ;-56]$} & {$\left[7.10^{-5} ; 2.10^{-3}\right]$} \\
\hline Dodecane & {$[-66 ;-51]$} & {$\left[1.10^{-4} ; 3.10^{-3}\right]$} \\
\hline Octyl acetate & {$[-69 ;-60]$} & {$\left[9.10^{-5} ; 1.10^{-4}\right]$} \\
\hline Methyl salicylate & {$[-90 ;-88]$} & {$\left[2.10^{-5} ; 3.10^{-5}\right]$} \\
\hline Glycerol carbonate & {$[-85 ;-79]$} & {$\left[3.10^{-5} ; 5.10^{-5}\right]$} \\
\hline Ethylene glycol & {$[-40 ;-32]$} & {$\left[3.10^{-5} ; 1.10^{-4}\right]$} \\
\hline
\end{tabular}


Example of image analysis to calculate the fibril diameters

The following example shows the results of the image analysis (thanks to ImageJ) for octyl acetate C18' organogel.

\begin{tabular}{|c|c|}
\hline $\mathrm{N}^{\circ}$ Fibril & $\begin{array}{c}\text { Diameter } \\
(\mu \mathrm{m})\end{array}$ \\
\hline 1 & 0.065 \\
\hline 2 & 0.064 \\
\hline 3 & 0.049 \\
\hline 4 & 0.068 \\
\hline 5 & 0.088 \\
\hline 6 & 0.068 \\
\hline 7 & 0.099 \\
\hline 8 & 0.087 \\
\hline 9 & 0.096 \\
\hline 10 & 0.07 \\
\hline
\end{tabular}

\begin{tabular}{|c|c|}
\hline $\begin{array}{c}\text { Mean Diameter } \\
(\mu \mathrm{m})\end{array}$ & $\begin{array}{c}\text { Standard } \\
\text { deviation }\end{array}$ \\
\hline 0.0754 & 0.0153 \\
\hline
\end{tabular}

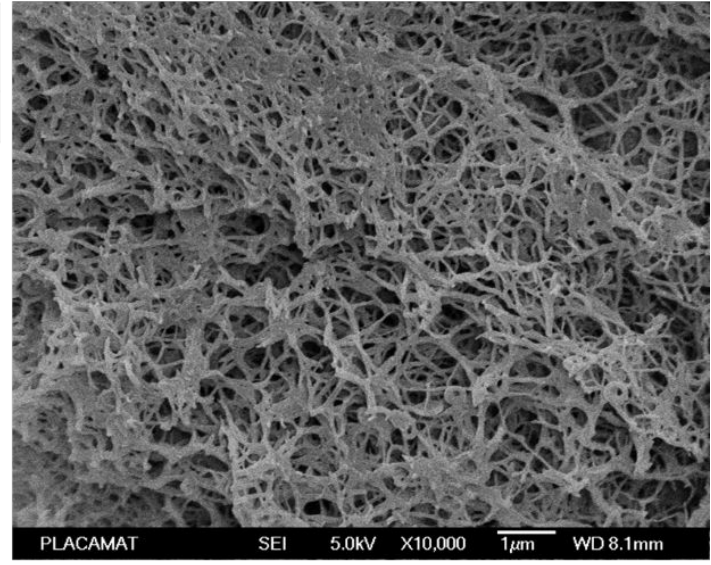

Figure S4: Example of fibrils diameter measurements on the C18' (2 wt\%) / octyl acetate organogel from a cryo-SEM picture.

\section{Rheological measurements}

\section{Gap contraction during the gelification kinetics}

The following table (Table 3 ) shows the gel contraction measured during the time ( $\Delta$ (gap)) and the corresponding elastic modulus $\left(G^{\prime}\right)$. No clear correlation between the gel modulus and the gel contraction was found.

Table S3: Gap variation during the kinetics and the corresponding elastic modulus of the gels of interest.

\begin{tabular}{|c|c|c|}
\hline Solvent & $\begin{array}{c}\Delta \text { (gap) } \\
\text { (mm) }\end{array}$ & $\mathrm{G}^{\prime}(\mathrm{Pa})$ \\
\hline o-xylene & 0.07 & $1.5 .10^{2}$ \\
\hline Dodecane & 0.19 & $3.10^{2}$ \\
\hline Octyl acetate & 0.05 & $1.10^{4}$ \\
\hline Methyl salicylate & 0.14 & $2.10^{4}$ \\
\hline Glycerol carbonate & 0.16 & $5.10^{4}$ \\
\hline
\end{tabular}

